Published in final edited form as:

Arthritis Care Res (Hoboken). 2017 December ; 69(12): 1863-1870. doi:10.1002/acr.23211.

\title{
Bone Mineral Density and the Risk of Hip and Knee Osteoarthritis: the Johnston County Osteoarthritis Project
}

\author{
Kamil E. Barbour, PhD ${ }^{1}$, Louise B. Murphy, PhD $^{1}$, Charles G. Helmick, MD ${ }^{1}$, Jennifer M. \\ Hootman, $\mathbf{P h D}^{1}$, Jordan B. Renner, $\mathbf{M D}^{3,4}$, and Joanne M. Jordan, MD MPH ${ }^{2,4}$ \\ ${ }^{1}$ Arthritis Program, Division of Population Health, CDC, Atlanta, GA, USA \\ ${ }^{2}$ Department of Medicine, University of North Carolina, Chapel Hill, NC, USA \\ ${ }^{3}$ Department of Radiology, University of North Carolina, Chapel Hill, NC, USA \\ ${ }^{4}$ Thurston Arthritis Research Center, University of North Carolina, Chapel Hill, NC, USA
}

\section{Abstract}

Objectives-To address knowledge gaps regarding the relationship between bone mineral density (BMD) and incident hip or knee osteoarthritis (OA); specifically, lack of information regarding hip OA or symptomatic outcomes.

\begin{abstract}
Methods-Using data $(\mathrm{N}=1,474)$ from the Johnston County Osteoarthritis (JoCo OA) Project's first (1999-2004) and second follow-up (2005-2010) of participants aged $\_45$ years we examined the association between total hip BMD and both hip and knee OA. Total hip BMD was measured using dual-energy X-ray absorptiometry, and participants were classified into sex-specific quartiles (low, intermediate low, intermediate high, and high). Radiographic osteoarthritis (ROA) was defined as development of Kellgren-Lawrence grade $\geq 2$. Symptomatic ROA (sROA) was defined as onset of both ROA and symptoms. Weibull regression modeling was used to estimate hazard ratios (HR) and 95\% confidence intervals (95\% CIs).
\end{abstract}

\begin{abstract}
Results-Median follow-up time was 6.5 (range=4.0-10.2) years. In multivariate models, and compared with participants with low BMD, those with intermediate high and high BMD were less likely to develop hip sROA (HR (95\% CIs) $0.52(0.31-0.86)$ and $0.56(0.31-0.86)$, respectively; p-trend $=0.024)$; high BMD was not associated $(0.69(0.45-1.06))$ with risk of hip ROA.

Compared with participants with low BMD, those with intermediate low and intermediate high total hip BMD were more likely to develop knee sROA (2.15 (1.40-3.30) and 1.65 (1.02-2.67), respectively; $\mathrm{p}$-trend $=0.325)$; similar associations were seen with knee ROA.
\end{abstract}

Conclusions-Our findings suggest that higher BMD may reduce the risk hip sROA, while intermediate levels may increase the risk of both knee sROA and ROA.

\footnotetext{
Corresponding Author: Kamil Barbour, Centers for Disease Control and Prevention, 4770 Buford Highway MS\#F78, Atlanta, GA, 30341, iyk1@cdc.gov, Phone: 770-488-5145.

Publisher's Disclaimer: This is a PDF file of an unedited manuscript that has been accepted for publication. As a service to our customers we are providing this early version of the manuscript. The manuscript will undergo copyediting, typesetting, and review of the resulting proof before it is published in its final citable form. Please note that during the production process errors may be discovered which could affect the content, and all legal disclaimers that apply to the journal pertain.

CDC Disclaimer: The findings and conclusions in this report are those of the authors and do not necessarily represent the official position of the Centers for Disease Control and Prevention
} 


\section{Keywords}

bone mineral density; radiographic and symptomatic knee and hip osteoarthritis; incidence; Weibull regression; interval censored data

\section{Introduction}

Both knee and hip osteoarthritis (OA) are common chronic conditions in the U.S. In a systematic review(1), the prevalence of knee and hip radiographic OA (ROA) among adults aged $\geq 45$ years was $19.2 \%$ and $27.8 \%$ for knee ROA, and $27.0 \%$ for hip ROA. Furthermore, the prevalence of knee and hip symptomatic ROA (sROA) in this population was 6.7 to $16.7 \%$ for knee sROA and $9.2 \%$ for hip sROA. Both OA of the knee and hip can be particularly disabling $(2,3)$, and may lead to reduced quality of life(4), early retirement(5), expensive joint replacement $(155,000 \mathrm{OA}$-attributable knee replacements and 65,550 OAattributable hip replacements in the U.S. in 2010)(6), and are linked to an increased risk of mortality. $(7,8)$

Higher bone mineral density (BMD), particularly of the hip, has been shown to reduce the risk of fractures in both men and women. $(9,10)$ This lower fracture risk may occur at the expense of an increased risk of incident knee ROA in middle-aged and older adults.(11-13) The biological mechanism wherein BMD may influence OA risk has not been established and prior statistically significant findings may result from not controlling for unmeasured confounders (e.g., genetic(14), bone morphology(15), skeletal growth factors(16), and bone geometry $(17,18))$. Furthermore, less is known about the relationship of BMD and incident sROA, which is an outcome with greater clinical and public health relevance. Additionally, to our knowledge, the association between BMD and incident hip ROA or sROA has not been examined.

To address these knowledge gaps, we examined the association between total hip BMD and incident hip and knee ROA and sROA among middle age and older ( $\geq 45$ years) communitydwelling white and black Johnston County Osteoarthritis (JoCo OA) Project participants.

\section{Patients and Methods}

\section{Study Population}

The JoCo OA Project is an ongoing population-based prospective cohort study of knee and hip OA in Johnston County, North Carolina (NC). Sampling methods are described elsewhere.(19) From May 1991 to December 1997, the project recruited 3,068 communitydwelling, non-institutionalized black and white participants, aged 45 years or older, who were physically and mentally capable of completing the project protocol and had resided in one of 6 townships in Johnston County, NC for at least 1 year. The study was approved by the Institutional Review Boards of the University of North Carolina Schools of Medicine and Public Health and the Centers for Disease Control and Prevention. All participants gave written informed consent at the time of recruitment. 
BMD measurements were added to the protocol at first follow-up (T1/ T1*; 1999-2004). Thus, for our study, the baseline occurred at T1/T1*, when 2,573 participants completed both a home and clinic visit; 2,065 of these participants had a BMD measurement (Figure). The $\mathrm{T} 1 *$ participants were an enrichment cohort enrolled at the first follow-up in the JoCo OA Project (T1*, 2003-2004) to supplement the T1 cohort. At the subsequent follow-up visit (T2; 2005-2010), 591 (28.6\%) of these 2,065 participants had died, moved away, become physically or mentally unable to complete the protocol, or were otherwise lost to follow-up, resulting in 1,474 participants with clinical measurements at both visits. (Figure). The median follow-up time (from $\mathrm{T} 1 / \mathrm{T} 1 *$ to $\mathrm{T} 2$ ) of 1,474 participants was 6.5 years (range 4.0-10.2 years).

\section{BMD Measurement}

For BMD we used total hip areal BMD (aBMD) $\left(\mathrm{g} / \mathrm{cm}^{2}\right)$, which was measured using Dual Energy X-ray Absorptiometry (DXA) (Hologic QDR Delphi A, Bedford, MA, USA) scans. To avoid redundancy we did not include femoral neck aBMD (a large component of total hip BMD), which is highly correlated with total hip BMD. We did not consider lumbar spine aBMD, because of its discordance with hip aBMD and its lower clinical utility [excluded from FRAX (a tool used to estimate 10-year probability of fracture)].(20) We calculated sexspecific aBMD quartiles because there were large differences in the mean distribution of aBMD by sex [men: mean (SD) aBMD $=0.996(0.165)$ and women: mean (SD) $\mathrm{aBMD}=0.878$ (0.158), $\mathrm{t}$-test $=15.867$, $\mathrm{p}$-value $<0.001]$. Those in the top quartile $(\mathrm{Q} 4)$ for aBMD were considered to have high aBMD, while those in the bottom quartile (Q1) were classified as having low aBMD. Participants with aBMD measurements in either quartiles 2 $(\mathrm{Q} 2)$ or $3(\mathrm{Q} 3)$ were classified as having intermediate low and intermediate high BMD, respectively.

\section{OA Outcomes}

A single bone and joint radiologist (JBR) performed posteroanterior paired readings of knee and hip radiographs at baseline and follow-up using the conventional Kellgren/Lawrence (K/L) scale.(21) The order of the radiographs was not known to the radiologist. Previous interrater and intrarater reliability were high (weighted $\kappa=0.86$ and 0.89 , respectively).(19) We examined 4 OA outcomes: hip ROA hip sROA, sROA knee ROA, and knee sROA (Figure). We defined incident ROA as K/L grade $\geq 2$ or joint replacement at follow-up in a joint with $\mathrm{K} / \mathrm{L}$ grade $<2$ at baseline. We excluded participants with any of the following in the relevant joint at study baseline: $\mathrm{K} / \mathrm{L}$ grade $\geq 2$, a joint replacement, missing radiographic data, or a non-OA diagnosis (i.e., radiographic evidence of inflammatory arthritis [e.g., rheumatoid arthritis]). We defined incident sROA as a joint with ROA and symptoms at follow-up among participants without both ROA and symptoms in the same joint at baseline. This definition of sROA has been accepted and used extensively in the OA literature.(22-26) We defined joint symptoms as a "yes" answer to the clinic survey question "on most days, do you have pain, aching or stiffness, in your hip/knee," asked separately for the left and right joints. Analytic sample sizes for symptomatic outcomes were greater than for radiographic outcomes, because participants assessed for the symptomatic outcomes had to have both symptoms and radiographic evidence of $\mathrm{OA}$ in the same joint to be excluded at baseline (Figure). 


\section{Potential Confounders}

We included several potential confounders of the BMD/OA relationship because they have been shown to be associated with BMD and OA, but are not in the causal pathway. We used study baseline values for: self-reported age, sex, race, education (no college versus attended college), body mass index (BMI), physical activity (meeting physical activity guidelines), smoking history, history of knee injury (injury at any knee joint) for knee OA outcomes analyses, history of hip injury (injury at any hip joint) for hip OA outcome analyses, history of bisphosphonate use, and history of steroid use. Physical activity was measured using the Minnesota Leisure Time Physical Activity (MLTPA) questionnaire, which collects selfreported information on physical activity during the previous year. (27) A previous comprehensive evaluation of the MLTPA concluded that the validity and reliability of this instrument were reasonably good.(28) We used the frequency and duration of 62 activities classified as moderate or vigorous activities to estimate the average minutes of moderateequivalent physical activity per week. Participants were grouped into two categories: those who met vs. those who did not meet the HHS physical activity guidelines of $\geq 150$ minutes of moderate-equivalent physical activity per week.(29) History of bisphosphonate use was defined as a "yes" response to the question "have you used any of the following for any reason - bisphosphonates (such as Fosamax and Didronel)"? History of steroid use was defined as a "yes" response to the question "have you taken any of the following for any reason - steroids (PO, IV, 1M, Inhaled)"? BMI was calculated from measured weight and height using the formula weight $(\mathrm{kg}) / \mathrm{height}^{2}\left(\mathrm{~m}^{2}\right)$ and was analyzed as a continuous variable.

\section{Statistical Analysis}

The unit of analysis was the person and an incident outcome needed to occur in only one knee or hip to be considered an event for that person. Differences in baseline characteristics by aBMD quartile were examined using chi-square and ANOVA statistical tests. Because the semi-parametric Cox regression model used in survival analysis is unable to accommodate interval-censored data (i.e., outcome has occurred within a time interval, but exact time of event is unknown), we used the Weibull parametric regression modeling to estimate hazard ratios (HRs) with 95\% confidence intervals (CIs).(30) Additional rationale for using Weibull Regression is described elsewhere.(25)

We performed multivariate analyses and checked for interactions by sex, but the interaction terms were not significant, so we only report findings overall and not stratified by sex. We compared participants in high, intermediate high, and intermediate low aBMD quartiles to those with low aBMD. We performed a test of trend across aBMD quartiles to examine whether a dose-response existed between $\mathrm{aBMD}$ and incident $\mathrm{OA}$. All multivariate analyses adjusted for 9 potential confounders: age, sex, race, BMI, education, physical activity, smoking history, history of bisphosphonate use, and history of steroid use. For knee OA outcomes we included prior history of knee injury; for hip OA outcomes we included prior history of hip injury. We performed a sensitivity analysis excluding participants undergoing arthroplasty at follow-up. We performed this analysis for those without hip or knee sROA at baseline. We were precluded from performing this sensitivity analyses for the participants 
without knee ROA or hip ROA at baseline, because of low incidence of arthroplasty in these two groups (0 knee replacements and 2 hip replacements).

To better understand how aBMD may be associated with hip or knee OA, we performed a secondary analysis to examine the association between aBMD and individual radiographic features in adults without knee or hip ROA. Osteophytes and joint space narrowing (JSN) were graded 0-3 for severity based on the Burnett et al Radiographic Atlas of OA.(31) JSN and osteophyte worsening in the knee were defined as at least a 1-grade increase in either the medial or lateral compartment of the tibiofemoral joint at follow-up. We defined hip JSN and osteophyte worsening similarly, but with JSN measured at the superior, axial, or medial(32); and osteophytes measured at the medial or lateral acetabular and/or femoral part of the joint. Participants were included in the secondary analysis if they had a K/L grade $<2$ at baseline. For instance, a participant could have evidence of osteophytes at baseline, but not meet $\mathrm{K} / \mathrm{L}$ criteria.

We used sampling weights to address the complex sample design of the JoCo Project (25) and generalize findings to the Johnston County population. We set the significance level at $\mathrm{p}<0.05$ for two tailed tests. We performed all analyses using Stata 14.0 software (Stata Corp., College Station, TX, USA), and used the Stata validated module INTCENS to perform the interval censored survival analysis.(33)

\section{Results}

At our study baseline (T1/T1*), there were 975 and 928 adults without knee ROA (KL<2) and hip ROA (KL $<2$ ), respectively (Figure). The number who progressed to $\mathrm{KL} \geq 2$ for those without knee ROA and hip ROA were 245 and 251, respectively. The cumulative incidence (proportion of new OA cases among study participants of hip ROA, hip sROA, knee ROA, and knee sROA was $27.0 \%, 9.9 \%, 25.1 \%$ and $13.2 \%$, respectively. At baseline and among those without hip ROA, 740 adults had JSN=0 and 188 had JSN=1, and 143, 739, and 46 had an osteophyte grade of 0,1 , and 2, respectively. At baseline and among those without knee ROA, 890 adults had JSN=0 and 85 had JSN=1. The number of adults who progressed at the hip for JSN and osteophytes were 95 and 227, respectively (Supplementary Table 1). The number of adults who progressed at the knee for JSN and osteophytes were 254 and 434, respectively (Supplementary Table 2). Compared with those with low aBMD, participants with high aBMD, were younger (mean age $=65.3$ and 56.6 years, respectively), more likely to be black (10.0\% and $44.0 \%$, respectively), have higher BMI (26.7 and 34.1 $\mathrm{kg} / \mathrm{m}^{2}$, respectively), meet physical activity recommendations ( $49.8 \%$ and $55.7 \%$, respectively) and have a history of steroid use (22.5\% and $34.6 \%$, respectively), and less likely to attend college ( $25.3 \%$ and $17.6 \%$, respectively), be former smokers (30.6\% and $20.5 \%$, respectively), and have a history of bisphosphonate use $(9.1 \%$ and $2.5 \%$, respectively). There was no association between aBMD and history of knee or hip injury (Table 1).

\section{Multivariate Analysis for Hip OA: Risk of Hip ROA and sROA}

Hip ROA-Compared with adults with low aBMD, those with intermediate low (HR: 0.72; 95\% CI: 0.51, 1.03), intermediate high (HR: 0.87; 95\% CI: 0.58, 1.31), and high (HR: 0.69; 
95\% CI: 0.45-1.06) aBMD were no more likely to develop incident hip ROA (Table 2). The risk of hip ROA did not decrease with increasing levels of aBMD (p-trend=0.215).

Hip sROA-Those with high (HR: 0.56; 95\% CI: 0.31-0.86) and intermediate high (HR: 0.52; 95\% CI: 0.31-0.86) aBMD were significantly less likely to develop incident hip sROA than those with low aBMD (Table 2). There was an inverse dose-response relationship: incident hip sROA decreased with rising aBMD (p-trend=0.024). Excluding incident hip replacements as an outcome in the sensitivity analysis did not change the dose-response relationship between aBMD and hip sROA; however, those with high aBMD no longer had a reduced significant risk of hip sROA, although this association was borderline (Table 2).

Hip JSN and Osteophyte Worsening-There were no significant associations between aBMD and hip JSN or osteophyte worsening (Supplementary Table 1), although there was an elevated (greater than 60\%) borderline-significant risk of osteophyte worsening among adults with intermediate low (HR: 1.61; 95\% CI: 0.998-1.57) and high aBMD (HR: 1.50; 95\% CI: 0.98-2.85), p-trend $=0.08$.

\section{Multivariate Analysis Knee OA: Risk of Knee ROA and sROA}

Knee ROA-Those with intermediate low (HR: 1.57; 95\% CI: 1.07-2.15) and intermediate high aBMD (HR: 1.64; 95\% CI: 1.14-2.36) were significantly more likely to develop incident knee ROA than those with low aBMD, however, there was no linear dose-response relationship between aBMD and incident knee ROA ( $\mathrm{p}=0.120)$ (Table 3$)$.

Knee sROA-Those with intermediate low (HR: 2.15; 95\% CI: 1.40-3.30) and intermediate high (HR: 1.65 ; 95\% CI: 1.02-2.67) aBMD were significantly more likely to develop incident knee sROA than those with low aBMD, however, there was no doseresponse relationship between aBMD and incident knee sROA ( $\mathrm{p}=0.325)$ (Table 3$)$. Excluding incident knee replacements as an outcome slightly attenuated the association between BMD and knee sROA; having intermediate high BMD was no longer associated with an increased risk of knee sROA (Table 3). The dose-response relationship between aBMD and knee sROA remained non-significant after excluding incident knee replacements in the sensitivity analysis as an outcome. Furthermore, having intermediate high aBMD was no longer associated with an increased risk of knee sROA (Table 3).

Knee JSN and Osteophyte Worsening-There was one significant association between aBMD and knee JSN, but no associations with worsening osteophytes (Supplementary Table 2). Adults with intermediate high aBMD were significantly more likely to develop knee JSN (HR: 1.50; 95\% CI: 1.05-2.13) compared with adults with low aBMD.

\section{Discussion}

Higher aBMD was significantly associated with a lower risk of hip sROA among adults age $\geq 45$ years in Johnston County, NC. Furthermore, intermediate, but not high, levels of aBMD were associated with an increased risk of both knee ROA and sROA. 
To our knowledge, this is the first study to evaluate the association between aBMD and incident hip OA. Those with high aBMD had a reduced risk of $44 \%$ for hip sROA, compared with those with low aBMD, and there was a dose-response relationship showing that greater aBMD was associated with lower risk of hip sROA. Three prior cross-sectional studies reported contrasting results, showing significantly higher aBMD and volumetric BMD (vBMD) among those with hip ROA (34-36), but they differ from our results in at least 2 important ways. First, these studies were cross-sectional, whereas our study looked at associations over time. Second, these studies used different definitions of hip OA (e.g., the Croft grading scale of $\geq 1$ to define hip OA(37), which only required a case to have osteophytosis [23]). Interestingly, we found an elevated risk (albeit marginally nonsignificant) between greater aBMD and osteophyte worsening, and no association with JSN, which was mostly consistent with the aforementioned studies (34-36). Thus, the prior crosssectional link between higher BMD and greater prevalence of hip OA appears to be attributable to osteophytes and not JSN. From a clinical perspective, these results imply that patients can maintain a healthy BMD, which is necessary to lower their fracture risk, without the increased risk of developing hip OA.

Adults with intermediate levels of aBMD had an increased risk of knee ROA and sROA, but high aBMD levels were not associated, and there were no significant dose-response relationships, suggesting a non-linear association. The Framingham Study reported similar findings of a p-trend for BMD quartiles of 0.222 and an odds ratio of $(\mathrm{OR})=2.2$ (95\% CI: 0.8-6.6) for participants in the high aBMD category (38). The Baltimore Longitudinal Study of Aging failed to find an association $(\mathrm{OR}=0.78(95 \% \mathrm{CI}, 0.51,1.20)$ between femoral neck aBMD (the largest component of hip aBMD) and incident knee OA (measured using K/L grade 2 ) for each standard deviation increase in aBMD) (39). Our findings regarding individual radiographic features showed an increased risk of JSN, consistent with the MOST study.(11) In contrast with other studies, we did not find a link between higher aBMD and knee osteophyte worsening $(11,13,38)$. Because we showed that intermediate BMD increased the risk of incident knee OA independent of many other potential confounders and risk factors for knee OA, our findings have clinical relevance for identifying adults at highest risk. We acknowledge that these associations may, however, be explained by factors not controlled for in the analysis (e.g., bone quality, morphology, and genetics).

Strengths of this study included 1) a large racially/ethnically diverse population-based sample that is generalizable to a community population, 2) adjustment for many potential confounders (e.g., age, sex, race, BMI, education, previous hip or knee joint injury, bisphosphonate use, and steroid use), 3) the longitudinal study design, and 4) management of interval censoring with Weibull regression modeling. A logistic or binomial regression model would not have accounted for time in the analysis. Furthermore, the use of a Cox regression model with the midpoint time at follow-up would result in largely inaccurate HR estimates when time intervals vary greatly.(40)

There were also limitations to this study. First, we did not have sufficient statistical power to assess the association between aBMD and incident sROA when applying a more restrictive case definition (e.g., limiting sROA to only those who developed it in a joint without both symptoms and ROA at baseline). Second, only 3.2\% met the World Health Organization 
definition of osteoporosis (BMD T-score less than 2.5 deviations below the mean of healthy woman), and thus, we were unable to examine the effect of osteoporosis on development of knee or hip OA. Third, attrition bias may have occurred because JoCo participants who completed the T2 follow-up have been shown to differ in some characteristics (e.g., younger age, more likely to be female or white) from those who did not complete the follow-up.(25) Assessing the direction of the attrition bias (if any) is not possible, because we did not have OA status at follow-up for the missing participants. Fourth, the incidence of OA in certain aBMD groups was low, which impacted the precision of some of our estimates. Fifth, although we adjusted for many covariates, residual confounding is a component of all observational studies. For instance, we did not account for the genetic polymorphisms that may be linked with high BMD and development of OA $(36,41,42)$, Genetic factors play a large role in hip OA etiology by accounting for about $60 \%$ of the explained variance(43), and are not controlled for in this analysis. Furthermore, elevated levels of skeletal growth factors are linked to high BMD and could lead to osteophyte formation (16), bone shape (e.g., a flatter superior aspect of femoral head and a more curved medial aspect of the femoral head) $(44,45)$, bone marrow lesion size (46), and measures of bone geometry(17, 18) (e.g., greater cross-sectional area, femoral neck width, and cross-sectional moment of inertia). Sixth, aBMD partially accounts for the variance in bone geometry, because of the two-dimensional cross-sectional areal measurement of BMD. A more accurate assessment of bone density can be provided by vBMD, which accounts for a 3-dimensional measurement of bone volume (i.e., bone depth in addition to cross-sectional area). Future studies should evaluate the association between BMD and incident OA using vBMD. Seventh, some of the covariates were assessed via self-report and are subject to recall bias (e.g., physical activity). Finally, the question on steroid use is generic and likely captures both catabolic and anabolic steroids (e.g., testosterone-like steroids), which have different impacts on BMD.

In conclusion, high total hip aBMD was associated with a decreased risk of hip sROA among middle-aged and older community-dwelling adults followed for a median of 6.5 years after adjusting for multiple confounders. Additionally, there was a significant inverse dose-response relationship between aBMD and incident hip sROA. Intermediate but not high aBMD was associated with an increased risk of knee OA. Our findings suggest that having high aBMD does not increase the risk of hip or knee OA and may reduce the risk of hip sROA. Additional studies examining hip OA and the symptomatic OA outcomes are needed to confirm these initial findings.

\section{Supplementary Material}

Refer to Web version on PubMed Central for supplementary material.

\section{Acknowledgments}

Funding: "Cooperative agreements S043 and S3486 from the Centers for Disease Control and Prevention, and the National Institute of Arthritis and Musculoskeletal and Skin Diseases (NIAMS) grant numbers: 5-P60-AR-30701 and 5-P60-AR49465." 


\section{References}

1. Lawrence RC, Felson DT, Helmick CG, Arnold LM, Choi H, Deyo RA, et al. Estimates of the prevalence of arthritis and other rheumatic conditions in the United States: Part II. Arthritis \& Rheumatism. 2008; 58(1):26-35. [PubMed: 18163497]

2. Guccione AA, Felson DT, Anderson JJ, Anthony JM, Zhang Y, Wilson P, et al. The effects of specific medical conditions on the functional limitations of elders in the Framingham Study. American journal of public health. 1994; 84(3):351-8. [PubMed: 8129049]

3. Felson DT, Lawrence RC, Dieppe PA, Hirsch R, Helmick CG, Jordan JM, et al. Osteoarthritis: new insights. Part 1: the disease and its risk factors. Annals of internal medicine. 2000; 133(8):635-46. [PubMed: 11033593]

4. Salaffi F, Carotti M, Stancati A, Grassi W. Health-related quality of life in older adults with symptomatic hip and knee osteoarthritis: a comparison with matched healthy controls. Aging clinical and experimental research. 2005; 17(4):255-63. [PubMed: 16285189]

5. Karpansalo M, Manninen P, Kauhanen J, Lakka TA, Salonen JT. Perceived health as a predictor of early retirement. Scandinavian journal of work, environment \& health. 2004:287-92.

6. Steiner C, Andrews R, Barrett M, Weiss A. HCUP Projections: mobility/orthopedic procedures 2011 to 2012. HCUP projections report. 2012; 3:2012-03.

7. Barbour KE, Lui LY, Nevitt MC, Murphy LB, Helmick CG, Theis KA, et al. Hip osteoarthritis and the risk of all-cause and disease-specific mortality in older women: Population-based cohort study. Arthritis \& Rheumatology. 2015

8. Liu Q, Niu J, Huang J, Ke Y, Tang X, Wu X, et al. Knee osteoarthritis and all-cause mortality: the Wuchuan Osteoarthritis Study. Osteoarthritis and Cartilage. 2015

9. Stone KL, Seeley DG, Lui LY, Cauley JA, Ensrud K, Browner WS, et al. BMD at multiple sites and risk of fracture of multiple types: long-term results from the Study of Osteoporotic Fractures. Journal of Bone and Mineral Research. 2003; 18(11):1947-54. [PubMed: 14606506]

10. Cummings SR, Cawthon PM, Ensrud KE, Cauley JA, Fink HA, Orwoll ES. BMD and risk of hip and nonvertebral fractures in older men: a prospective study and comparison with older women. Journal of Bone and Mineral Research. 2006; 21(10):1550-6. [PubMed: 16995809]

11. Nevitt MC, Zhang Y, Javaid MK, Neogi T, Curtis JR, Niu J, et al. High systemic bone mineral density increases the risk of incident knee OA and joint space narrowing, but not radiographic progression of existing knee OA: the MOST study. Annals of the rheumatic diseases. 2010; 69(01):163-8. [PubMed: 19147619]

12. Bergink A, Uitterlinden A, Van Leeuwen J, Hofman A, Verhaar J, Pols H. Bone mineral density and vertebral fracture history are associated with incident and progressive radiographic knee osteoarthritis in elderly men and women: the Rotterdam Study. Bone. 2005; 37(4):446-56. [PubMed: 16027057]

13. Hart DJ, Cronin C, Daniels M, Worthy T, Doyle DV, Spector TD. The relationship of bone density and fracture to incident and progressive radiographic osteoarthritis of the knee: the Chingford Study. Arthritis \& Rheumatism. 2002; 46(1):92-9. [PubMed: 11817613]

14. Yau MS, Yerges-Armstrong LM, Liu Y, Lewis CE, Duggan DJ, Renner JB, et al. Genome-wide Association Study of Radiographic Knee Osteoarthritis in North American Caucasians. Arthritis \& Rheumatology. 2016

15. Nelson AE, Golightly YM, Renner JB, Schwartz TA, Liu F, Lynch JA, et al. Variations in Hip Shape Are Associated with Radiographic Knee Osteoarthritis: Cross-sectional and Longitudinal Analyses of the Johnston County Osteoarthritis Project. The Journal of rheumatology. 2016; 43(2): 405-10. [PubMed: 26669914]

16. Hunter DJ, Spector TD. The role of bone metabolism in osteoarthritis. Current rheumatology reports. 2003; 5(1):15-9. [PubMed: 12590880]

17. Ding C, Cicuttini F, Jones G. Tibial subchondral bone size and knee cartilage defects: relevance to knee osteoarthritis. Osteoarthritis and cartilage. 2007; 15(5):479-86. [PubMed: 17291789]

18. Javaid M, Lane N, Mackey D, Lui LY, Arden N, Beck T, et al. Changes in proximal femoral mineral geometry precede the onset of radiographic hip osteoarthritis: The study of osteoporotic fractures. Arthritis \& Rheumatism. 2009; 60(7):2028-36. [PubMed: 19565486] 
19. Jordan JM, Helmick CG, Renner JB, Luta G, Dragomir AD, Woodard J, et al. Prevalence of knee symptoms and radiographic and symptomatic knee osteoarthritis in African Americans and Caucasians: the Johnston County Osteoarthritis Project. The Journal of rheumatology. 2007; 34(1): 172-80. [PubMed: 17216685]

20. Leslie W, Lix L, Johansson H, Oden A, McCloskey E, Kanis J. Spine-hip discordance and fracture risk assessment: a physician-friendly FRAX enhancement. Osteoporosis international. 2011; 22(3):839-47. [PubMed: 20959961]

21. Kellgren J, Lawrence J. Radiological assessment of osteo-arthrosis. Ann Rheum Dis. 1957; 16(4): 494-502. [PubMed: 13498604]

22. Felson DT, Niu J, Clancy M, Sack B, Aliabadi P, Zhang Y. Effect of recreational physical activities on the development of knee osteoarthritis in older adults of different weights: the Framingham Study. Arthritis Care \& Research. 2007; 57(1):6-12. [PubMed: 17266077]

23. McAlindon TE, Wilson PW, Aliabadi P, Weissman B, Felson DT. Level of physical activity and the risk of radiographic and symptomatic knee osteoarthritis in the elderly: the Framingham study. The American journal of medicine. 1999; 106(2):151-7. [PubMed: 10230743]

24. Segal NA, Torner JC, Felson D, Niu J, Sharma L, Lewis CE, et al. Effect of thigh strength on incident radiographic and symptomatic knee osteoarthritis in a longitudinal cohort. Arthritis Care \& Research. 2009; 61(9):1210-7. [PubMed: 19714608]

25. Barbour K, Hootman J, Helmick C, Murphy L, Theis KA, Schwartz T, et al. Meeting Physical Activity Guidelines and the Risk of Incident Knee Osteoarthritis: A Population-Based Prospective Cohort Study. Arthritis care \& research. 2014; 66(1):139-46. [PubMed: 23983187]

26. Murphy LB, Moss S, Do BT, Helmick CG, Schwartz TA, Barbour KE, et al. Annual incidence of knee symptoms and four knee osteoarthritis outcomes in the Johnston County Osteoarthritis Project. Arthritis care \& research. 2016; 68(1):55-65. [PubMed: 26097226]

27. Taylor HL, Jacobs DR Jr, Schucker B, Knudsen J, Leon AS, Debacker G. A questionnaire for the assessment of leisure time physical activities. Journal of chronic diseases. 1978; 31(12):741-55. [PubMed: 748370]

28. Richardson MT, Leon AS, Jacobs DR Jr, Ainsworth BE, Serfass R. Comprehensive evaluation of the Minnesota Leisure Time Physical Activity Questionnaire. Journal of clinical epidemiology. 1994; 47(3):271-81. [PubMed: 8138837]

29. Physical Activity Guidelines Advisory Committee Report, 2008. Nutr Rev. 2009; 67(2):114-20. [PubMed: 19178654]

30. Odell PM, Anderson KM, D'Agostino RB. Maximum likelihood estimation for interval-censored data using a Weibull-based accelerated failure time model. Biometrics. 1992; 48(3):951-9. [PubMed: 1420849]

31. Burnett, S. A radiographic atlas of osteoarthritis. Pfizer; 1994.

32. Lanyon P, Muir K, Doherty S, Doherty M. Influence of radiographic phenotype on risk of hip osteoarthritis within families. Annals of the rheumatic diseases. 2004; 63(3):259-63. [PubMed: 14962959]

33. Griffin J. INTCENS: Stata module to perform interval-censored survival analysis. Statistical Software Components. 2005

34. Chaganti R, Parimi N, Lang T, Orwoll E, Stefanick M, Nevitt M, et al. Bone mineral density and prevalent osteoarthritis of the hip in older men for the Osteoporotic Fractures in Men (MrOS) Study Group. Osteoporosis international. 2010; 21(8):1307-16. [PubMed: 20101493]

35. Nevitt MC, Lane NE, Scott JC, Hochberg MC, Pressman AR, Genant HK, et al. Radiographic osteoarthritis of the hip and bone mineral density. Arthritis \& Rheumatism. 1995; 38(7):907-16. [PubMed: 7612040]

36. Antoniades L, MacGregor AJ, Matson M, Spector TD. A cotwin control study of the relationship between hip osteoarthritis and bone mineral density. Arthritis \& Rheumatism. 2000; 43(7):1450. [PubMed: 10902745]

37. CROFT P, COOPER C, WICKHAM C, COGGON D. Defining osteoarthritis of the hip for epidemiologic studies. American journal of epidemiology. 1990; 132(3):514-22. [PubMed: 2389755] 
38. Zhang Y, Hannan M, Chaisson C, McAlindon T, Evans S, Aliabadi P, et al. Bone mineral density and risk of incident and progressive radiographic knee osteoarthritis in women: the Framingham Study. The Journal of rheumatology. 2000; 27(4):1032-7. [PubMed: 10782833]

39. Hochberg MC, Lethbridge-Cejku M, Tobin JD. Bone mineral density and osteoarthritis: data from the Baltimore Longitudinal Study of Aging. Osteoarthritis and cartilage. 2004; 12:45-8.

40. Klein, JP., Moeschberger, ML. Survival analysis: techniques for censored and truncated data. Springer Science \& Business Media; 2003.

41. Boyden LM, Mao J, Belsky J, Mitzner L, Farhi A, Mitnick MA, et al. High bone density due to a mutation in LDL-receptor-related protein 5. New England Journal of Medicine. 2002; 346(20): 1513-21. [PubMed: 12015390]

42. Yerges-Armstrong LM, Yau MS, Liu Y, Krishnan S, Renner JB, Eaton CB, et al. Association Analysis of BMD-associated SNPs with Knee Osteoarthritis. Journal of Bone and Mineral Research. 2014; 29(6):1373-9. [PubMed: 24339167]

43. MacGregor AJ, Antoniades L, Matson M, Andrew T, Spector T. The genetic contribution to radiographic hip osteoarthritis in women. Arthritis Rheum. 2000; 43(11):2410-6. [PubMed: 11083262]

44. Lynch J, Parimi N, Chaganti R, Nevitt M, Lane N, Group SoOFR. The association of proximal femoral shape and incident radiographic hip OA in elderly women. Osteoarthritis and Cartilage. 2009; 17(10):1313-8. [PubMed: 19427402]

45. Nelson AE, Stiller J, Shi X, Leyland K, Renner JB, Schwartz TA, et al. Measures of hip morphology are related to development of worsening radiographic hip osteoarthritis over 6 to 13 year follow-up: the Johnston County Osteoarthritis Project. Osteoarthritis and Cartilage. 2015

46. Driban JB, Price LL, Lo GH, Pang J, Hunter DJ, Miller E, et al. Evaluation of bone marrow lesion volume as a knee osteoarthritis biomarker-longitudinal relationships with pain and structural changes: data from the osteoarthritis initiative. Arthritis Res Ther. 2013; 15(5):R112. [PubMed: 24020939] 


\section{Significance and Innovation}

- $\quad$ To our knowledge this is the first study to examine BMD and risk of hip OA as well as incident symptomatic OA outcomes.

- High BMD was associated with a lower risk of hip OA.

- Intermediate BMD levels were associated with an increased risk of knee OA. 


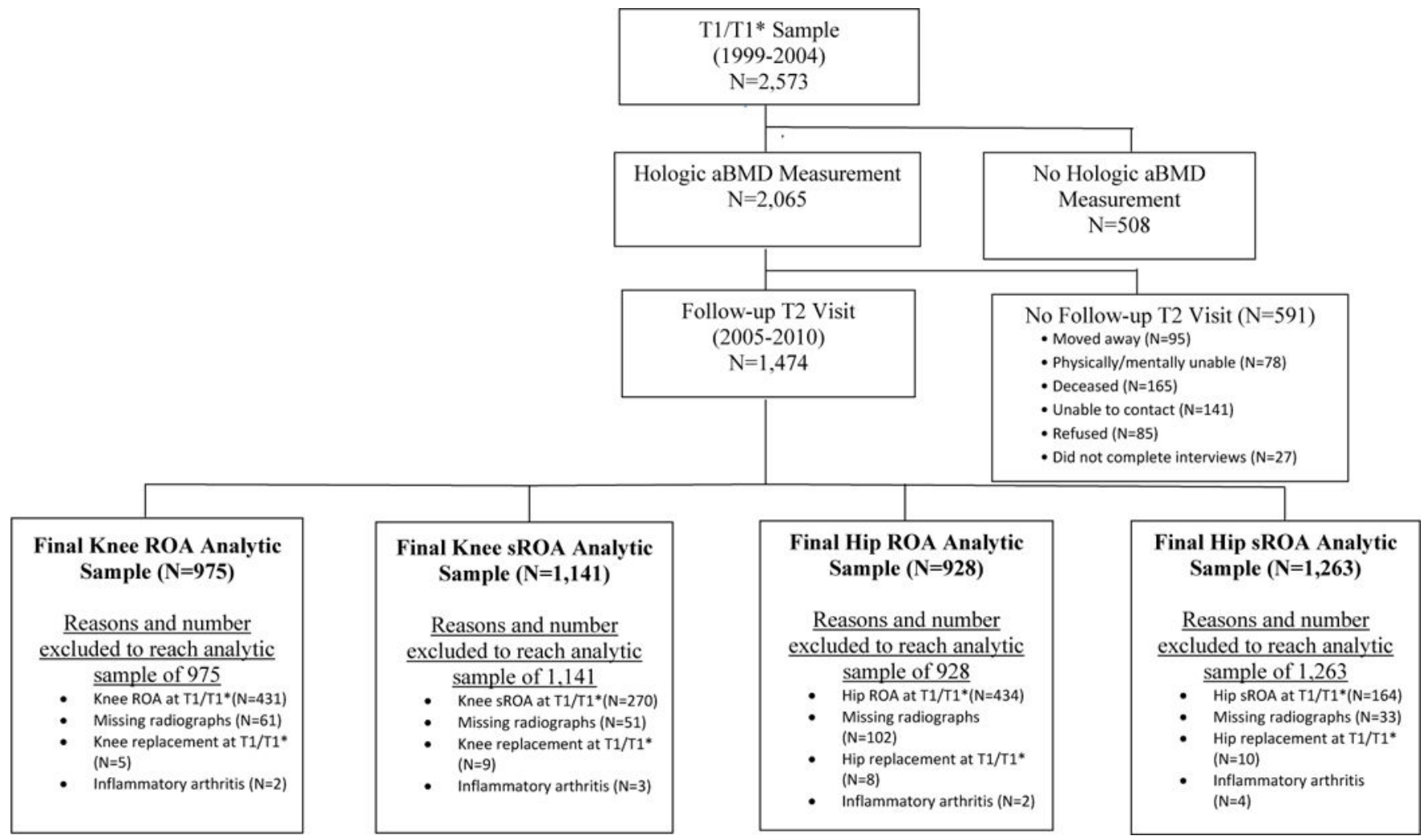

Figure.

Flow chart for the Johnston County Project participants from study baseline (T1 and T1* visits) to follow-up (T2) and the derivation of the 4 analytic samples 
Barbour et al.

Page 14

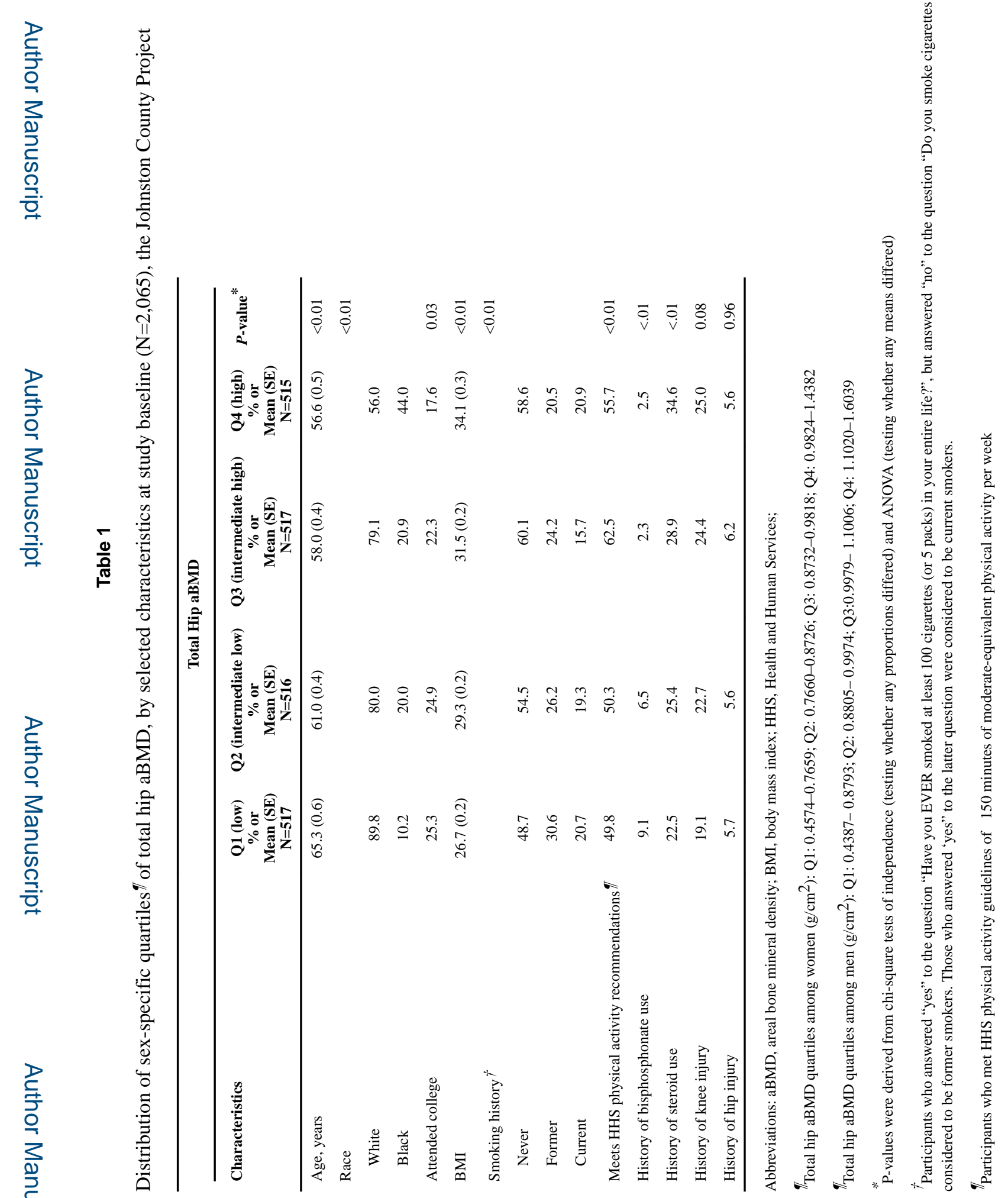

Arthritis Care Res (Hoboken). Author manuscript; available in PMC 2018 December 01. 


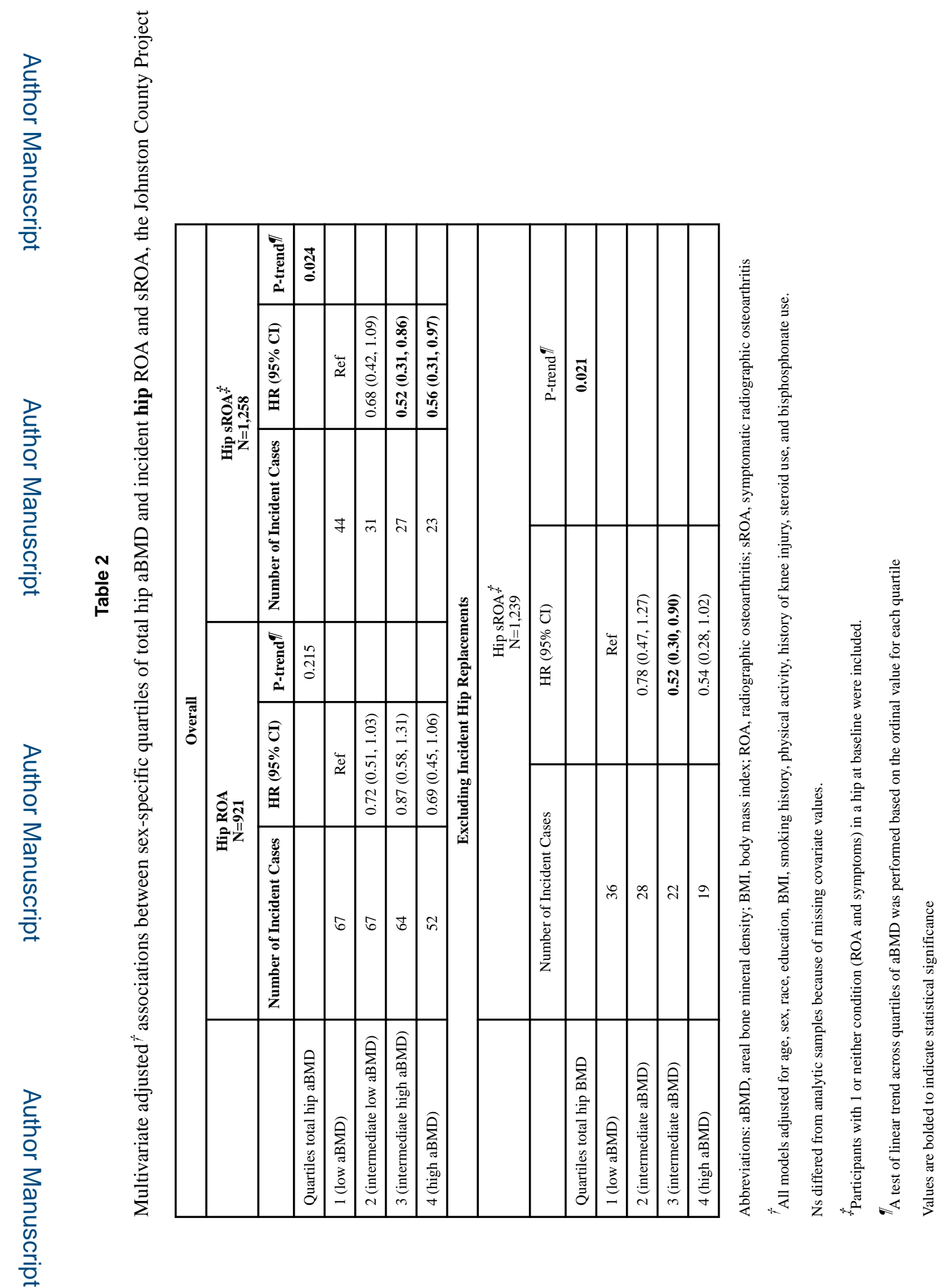

Arthritis Care Res (Hoboken). Author manuscript; available in PMC 2018 December 01. 
Barbour et al.

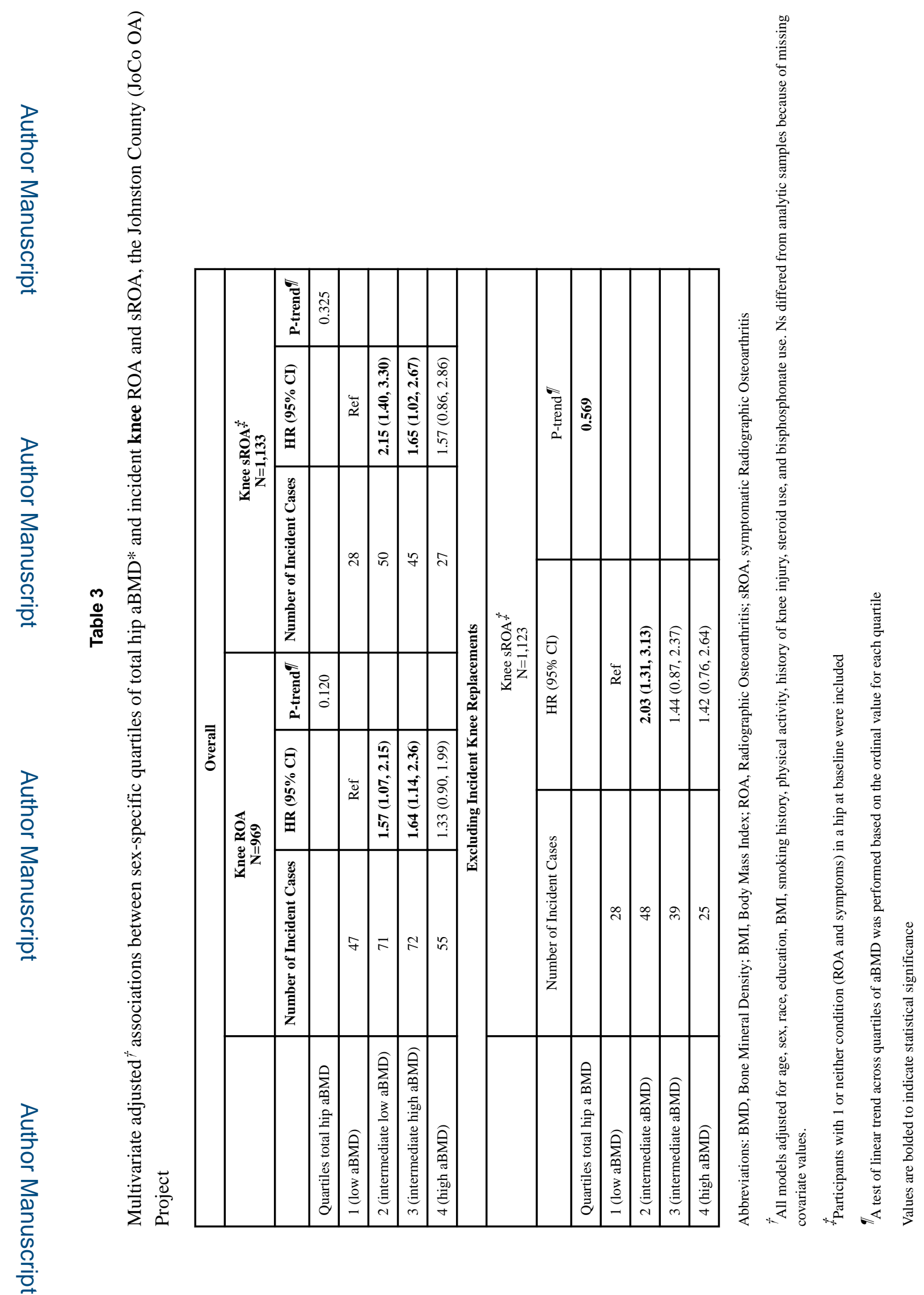

Arthritis Care Res (Hoboken). Author manuscript; available in PMC 2018 December 01. 